\title{
Aportes de las neurociencias para el desarrollo de estrategias de enseñanza y aprendizaje de las Matemáticas
}

\section{Contributions of Neuroscience to Develop Teaching Strategies and Learning of Mathematics}

\author{
Eddy Mogollón ${ }^{l}$ \\ Universidad Dr. Rafael Belloso Chacín \\ Maracaibo, Venezuela \\ eddymogollon@gmail.com \\ eddy.mogollon@urbe.edu
}

Recibido 27 de setiembre de 2010 • Aceptado 03 de noviembre de 2010

Resumen. El presente trabajo tiene como objetivo desarrollar algunas estrategias generadas a partir de las investigaciones en neurociencias. El propósito es contribuir con la enseñanza y aprendizaje de las Matemáticas. Se argumentó sobre la interrelación de la enseñanza compatible con el cerebro, así como sobre las estructuras cerebrales con el pensamiento en matemático. Se desarrollaron estrategias tomando en consideración niveles que abarcan lo cognitivo, semiótico, lenguaje, la afectividad y la superación de las fobias a la asignatura. La conclusión fundamental fue el imperante requerimiento educativo en el futuro próximo de un nuevo docente, cuya formación pedagógica debe incluir el conocimiento sobre la función cerebral, sus estructuras y sus implicancias con la educación, así como un cambio en la pedagogía y estructura currícular en la enseñanza de las Matemáticas.

Palabras clave. Estrategias, cerebro, neurociencias, Matemática.

Abstract. The goal of the present work is to develop some strategies based on research in neurosciences that contribute to the teaching and learning of mathematics. The interrelationship of education with the brain, as well as the relationship of cerebral structures with mathematical thinking was discussed. Strategies were developed taking into consideration levels that include cognitive, semiotic, language, affect and the overcoming of phobias to the subject. The fundamental conclusion was the imperative educational requirement in the near future of a new teacher, whose pedagogic formation must include the knowledge on the cerebral function, its structures and its implications to education, as well as a change in pedagogy and curricular structure in the teaching of mathematics.

Key words. Strategies, Brain, Neurosciences, Mathematics.

\footnotetext{
Doctorado en Ciencias de la Educación. Profesor Titular de la Universidad Dr. Rafael Belloso Chacín, Maracaibo, Zulia. Investigador de Planta del Centro de Investigación de Humanidades y Educación (CIHE/URBE). Profesor de Postgrado de la asignatura Estadística aplicada a la investigación. Profesor de Postgrado de la asignatura Análisis e Interpretación de reportes de investigación.
} 


\section{Introducción}

La enseñanza de las Matemáticas es una actividad compleja. Una gran cantidad de bibliotecas en el mundo están abarrotadas con teorías sobre su enseñanza y su aprendizaje. Disciplinas como epistemología, psicología cognoscitiva, semiótica, lingüística se alinean para dar soporte a tan importante disciplina. Se investiga sobre la relación de la sociedad, la historia, la cultura, la antropología con las Matemáticas y su aprendizaje. Es así como la enseñanza de las Matemáticas llega a tejer una malla interdisciplinar con otras ciencias, convirtiéndola en una ciencia compleja, pero emocionante para la investigación. Indudablemente, los eslabones de esa malla interdisciplinar están conformados por las ciencias pedagógicas.

Una de las disciplinas que hace presencia notable para impulsar la enseñanza y el aprendizaje de las Matemáticas es, sin duda, las neurociencias cognoscitivas. Aunque todavía hay autores que argumentan sobre la débil relación de las investigaciones neurocientíficas en el ámbito pedagógico, como soporte a las ciencias de la educación (Radford y André, 2009); otros como Salazar (2005) consideran que las neurociencias cognoscitivas y la educación matemática mantienen puntos de convergencia beneficiosos para ambas disciplinas.

En este sentido, ya se han realizado avances importantes en la investigación neurocientífica y la enseñanza de las Matemáticas. Investigadores como Beilock (2008), Edin, Klinberg, Johanssona, McNab, Tegne y Compte (2009), Ballestra, Martínez y Argibay (2006), Foss y Hadfield (1993), Goldin (1988), Op't, De Corte y Verschaffel (2001) y otros más, han realizado significativos estudios al respecto. Lo medular es que ya la neurociencia tiene un camino recorrido, y ha dado aportes a la educación en general y a la enseñanza de la Matemática en particular.

Se justifica el interés cada vez mayor de los investigadores neurocientíficos por la enseñanza de las Matemáticas, y también, los investigadores en didáctica de esta disciplina por los resultados obtenidos en las investigaciones neurocerebrales, dado que ambos sectores buscan esclarecer los problemas relacionados con la naturaleza del pensamiento matemático.

El objetivo de este trabajo es desarrollar algunas estrategias generadas a partir de las investigaciones en neurociencias para contribuir con la enseñanza y aprendizaje en las Matemáticas.

\section{Nuevos enfoques hacia una nueva ciencia en el aprendizaje matemático}

Es necesario mencionar que desde la segunda mitad del siglo XX hasta nuestros días, los avances mostrados por las ciencias de la educación en comprender el aprendizaje del ser humano son innegables. Sin embargo, todavía no se puede decir, con certeza, que estos avances se inserten con fundamento y rigor científico. Aún se está lejos de hacer predicciones y controlar los aprendizajes que se manifiestan en los estudiantes, así como de dotar de capacidad suficiente a docentes basados en resultados aislados del que enseña. He aquí un desafío a enfrentar las ciencias de la educación para este siglo (Waldegg, 2003).

Las teorías del aprendizaje han avanzado (caracterizando comportamientos, practicas, conductas) y han presentado algunas propuestas para la facilitación de la enseñanza y el aprendizaje. No obstante, muchas de estas propuestas se han apoyado en evidencias empíricas (limitadas) para un corto plazo, las cuales reducen las posibilidades de reproducir estas prácticas o eludir las indeseadas. La existencia de tan diversas teorías, para discernir sobre los mismos fenómenos, indudablemente, ha dado una clara muestra de incertidumbre en los enfoques presentados. 
Para Waldegg (2003), existe una nueva frontera, donde se vislumbran formas innovadoras de soportar las hipótesis sobre el aprendizaje, basadas en datos reales y puros, provenientes de los resultados obtenidos de acuerdo con el funcionamiento del cerebro. Según el razonamiento de esta investigadora, la psicología cognoscitiva debe interactuar e interrelacionarse con otras disciplinas para seguir aportando soluciones al aprendizaje. Pero la relación fundamental e ineludible que la psicología cognoscitiva debe afrontar, inmediatamente, es con la neurociencia. La neurociencia está aportando respuestas a situaciones de sumo interés para los docentes, por ejemplo; existen investigaciones donde se evidencia que tanto un cerebro en maduración como uno ya maduro se modifican estructuralmente cuando ocurre un evento de aprendizaje (Bransford, Brown y Cocking, 2003).

Desde la nueva expectativa en el estudio del cerebro, la emocionalidad vuelve a ocupar estadios importantes para el aprendizaje y, especialmente, en la enseñanza de las Matemáticas, colocando la racionalidad platónico-cartesianas en una disyuntiva epistémica. Para Coleman (2000), el aprendizaje no está separado de las emociones. Instruir a las personas en descubrir sus emociones es tan significativo para la enseñanza y el aprendizaje, como la instrucción en Matemáticas y la lecto-escritura.

\section{Neurociencias en el contexto de la educación matemática}

Es indudable que las neurociencias, como paradigma emergente, ha soportado, perfilado y en ocasiones, conceptualizado, al aprendizaje matemático, ya que ha aportado a las ciencias de la educación matemática un nuevo paradigma: El aprendizaje compatible con el cerebro. Es necesario recordar; sin embargo, que a pesar del desarrollo sostenido y confiable en los estudios del cerebro, incluyendo sus aportes al aprendizaje (o cognición), estos estudios se realizan en ambientes no educativos, controlados con equipos de alta tecnología y aunque no se pretende trasladar estos estudios al salón de clases, se debe reconocer, según Geake (2005); que si el aprendizaje es el móvil fundamental en la educación, entonces las investigaciones y descubrimientos generados a causa de la neurociencia, coadyuvan a comprender los procesos en el aprendizaje de los estudiantes, con la intención de enseñarles efectiva, placentera y adecuadamente. Para Wolfe (2001), un gran descubrimiento para la educación es la investigación relativa al cerebro, un campo extraño a los docentes hasta recientes fecha.

\section{El cerebro y las Matemáticas}

\section{Nuevos enfoques en la memoria de trabajo}

Una definición clásica, utilizada por muchos investigadores para definir la memoria de trabajo es la utilizada por Baddeley (1997), la cual menciona que es el sistema con un nivel funcional limitado, pero con capacidad para almacenar, manipular las informaciones, permitiendo así la ejecución de tareas cognitivas tales como el razonamiento, la comprensión y la resolución de problemas, soportadas por el mantenimiento y disponibilidad de esas informaciones. Agrega este autor que la memoria de trabajo es el espacio mental operativo, necesario para el recuerdo episódico, semántico, el pensamiento racional y la toma de decisiones. También, es útil para la comprensión del lenguaje, el cálculo y para todas las actividades del tipo cognitivo que requieran atención, así como el procesamiento controlado. 
Tal como define Baddeley, la memoria de trabajo asume un papel relevante para el cálculo y de acuerdo con los nuevos enfoques neurocientíficos, hay que considerarla, sobre todo, en el campo de la educación matemática. En este sentido, Edin et al. (2009), utilizando una simulación computacional, predicen que el entrenamiento activo de los lóbulos frontales mejora sustancialmente la capacidad en la memoria de trabajo. Sin embargo, esta actividad debe ser controlada, ya que un entrenamiento continuo estimula el incremento del número de receptores dopaminérgicos en la corteza cerebral, lo cual dificulta la memorización en plazos breves de tiempo. Esta investigación guía a un mejor entendimiento entre la interrelación de lo cognitivo y la estructura neuro-biológica del cerebro (Macnab, Varrone, Farde, Jucaite, Bystritsky, Forssberg, 2009).

Por otro lado, Morgado (2005) agrega que la memoria de trabajo utiliza la información recibida sensorialmente, para darle uso en la resolución mental de situaciones que requieran solución, para aplicarla en el acto de razonar o para la toma de decisiones. O sea, la memoria de trabajo es el umbral para un aprendizaje con significado y está muy estrechamente relacionada con la inteligencia del cada individuo.

Morgado concluye, también, que la memoria de trabajo se puede considerar un sistema generalizado de control cognitivo y ejecutivo, que dirige el comportamiento (aprendizaje) interrelacionando procesos mentales o neuronales como la atención, motivación, emoción y percepción. Este prestigioso neurocientífico, propone, sustituir el nombre regular de memoria de trabajo por el de sistema de cognición ejecutiva. Esto parece interesante en el contexto matemático, como se observará, cuando se trate más adelante sobre la atención y la emoción.

En el marco de las observaciones anteriores, la memoria de trabajo está ocupando cada día más espacios, reclamando un lugar privilegiado en la enseñanza y el aprendizaje. Un estudio significativo fue el realizado por la investigadora y sicóloga Beilock (2008), quien en su trabajo Math Performance in Stressful (Rendimiento en matemáticas bajo estrés), afirma que la memoria de trabajo es la responsable en la ansiedad matemática.

Para Beilock, siendo esta memoria la responsable de recibir información y retenerla para la toma de alguna decisión importante, esta información se consume o pierde en escenarios de ansiedad o estrés. Esta investigadora concluye que los individuos con alta capacidad en memoria de trabajo poseen niveles superiores en lo relativo a cálculo matemático; pero pierden, rápidamente, su memoria de trabajo en situaciones perturbadoras o de gran ansiedad. Lo anterior debe considerarse un punto muy importante para los docentes vinculados al dictado de asignaturas relacionadas con el cálculo matemático.

\section{Las Matemáticas y la atención}

En el aprendizaje, la atención siempre ha ocupado un papel fundamental. Pero la atención es un proceso sensible, oscilante y necesitada de la emoción, así como del descubrimiento de nuevas experiencias para que la atención se mantenga (Rosselló, 1998). Para Luria (1988), el tallo cerebral y el sistema activador reticular ascendente, denominado por las siglas SARA, son los que controlan los estados de alerta y vigilia necesarios para que se active la función atencional. La región frontocraneal, conjuntamente con el córtex límbico, son capaces de identificar un estimulo especifico y producir el bloqueo o inhibición de respuesta a estímulos posteriores.

Es importante, para el autor de este trabajo, mencionar este mecanismo, debido a que el sistema activador reticular ascendente representa un sistema neurofisiológico, el cual se manifiesta como un acto reflejo conocido por respuesta de orientación. Este se evidencia por manifestaciones 
del tipo vascular y motoras, como voltear la cabeza al objeto que causa interés, así como mantener una condición vigilante (alerta). También, surgen alteraciones en el sistema respiratorio y en el ritmo cardiaco (taquicardia), con consecuencias en el cese de toda actividad. Estos síntomas son expresiones inequívocas en cuanto a las reacciones que manifiestan muchos estudiantes ante la presencia de exámenes o materias con dificultad, en especial, las Matemáticas, tal como lo menciona Bauselas (2005), destacando el papel significativo que la atención y la emoción representan para la neurociencias cognitivas y la educación.

La atención es un proceso a nivel cognitivo fundamental. Esta puede dar acceso o negar la participación de estímulos sensoriales, tanto interna como externamente. La atención tiene muchas vías para manifestarse. Unas pueden ser por gestos, movimiento oculares, así como posturas corporales. Varios autores, por ejemplo; Sprenger (1999), Guisa, Riquelme y San Vicente (2007), Wolfe (2001); entre otros, mencionan que los neurotransmisores dopamina y norepinefrina (catecolaminas) son elementos claves en los procesos de vigilia, alerta, atención y concentración, así como en situaciones memorísticas o de motivación.

Haciendo una breve descripción de la activación en el encéfalo del neurotransmisor dopamina, puede decirse que inicia en el mesencéfalo, con proyecciones a otras áreas del telencéfalo, activando la motilidad del sujeto, también activa el sistema estriado-tálamo con respuesta en el movimiento. Por otro lado, en el núcleo accumbens esta sustancia activa aspectos como la emoción $\mathrm{y}$, en la corteza prefrontal, juega un papel fundamental en la toma de decisiones.

Es interesante observar la importancia de la activación dopaminérgica en la atención y el desempeño de tareas, sobre todo en Matemáticas. Un trabajo presentado por Volkow, Wang, Fowler, Telang, Maynard y Logan (2004) hace referencia a esto, al mencionar que el uso de psico-estimulantes dopaminérgicos (metilfenidato o d-anfetaminas) incrementan el desempeño y la motivación durante la práctica y resolución de tareas matemáticas, tanto en niños con déficit atencional, niños sanos e inclusive adultos; esto, sin riesgos de llegar a la psico-dependencia. Aunque el autor en este trabajo no comulga con la aplicación de estimulantes a niños sanos, es resaltante mencionar la significancia que podría tener la activación dopaminérgica en forma natural, es decir, a través de la emoción y motivación, para actividades de esta naturaleza.

Por otro lado, Wolkow et al. (2004) también determinaron que, en algunos sujetos, la privación del sueño les incrementó, considerablemente, el nivel de dopamina en las estructuras cerebrales denominadas: el estriatum, relacionado tanto con la motivación como con la recompensa y también el tálamo relacionado con la capacidad de alerta y vigilia. Estos investigadores, también, comprobaron que bajos niveles en dopamina se manifiestan como una sensación de fatiga, además del mal funcionamiento del cerebro en las tareas cognoscitivas.

El caso de la motivación en Matemáticas ha sido muy estudiado (Martínez y Galán, (2000); Covington, M. (2000). Sin embargo, un estudio reciente y de sumo interés es el trabajo presentado por Carmona et al. (2009), neurocientífica de la Universidad Autónoma de Barcelona, quien confirma que en el trastorno de atención e hiperactividad no solamente inciden la alteraciones cognitivas, sino también por déficit motivacional. De acuerdo con este hecho, concluye la investigadora, se puede explicar por qué los niños con trastorno por déficit de atención con hiperactividad poseen niveles atencionales desiguales, dependiendo del retardo/inmediatez para alcanzar la recompensa o placer.

Lo anterior induce, al autor, a pensar la relación de esta afirmación con una referencia teórica donde se incluyen similares palabras, tales como aprendizaje, estímulo, refuerzo, recompensa y respuesta. Esta referencia teórica que abarca las palabras anteriores es la teoría de aprendizaje denominada conductismo. ¿Será que la neurociencia está reivindicando al conductismo? Investigar esta situación seria de gran interés. 
Para la investigadora, es resaltante que un niño pueda estar en total concentración frente a un video juego durante muchas horas y no tenga la capacidad de estar atento a una clase o actividad matemática por diez minutos. Desde el punto de vista neurológico, se explica por alteraciones de áreas que involucran el núcleo accumbens. Esta área es necesaria para activar la motivación y, por consiguiente, la concentración y atención.

Uno de los elementos relevantes que afectan la atención es, indudablemente, la alimentación. Según Sprenger (1999), el consumo de proteínas es un potenciador que incide positivamente en la atención, ya que ella se provee de los aminoácidos necesarios para producir la norepinefrina y la dopamina. También se debe tener en consideración que la mayor intensidad neuroquímica (transmisión sináptica) se alcanza en la mañana, cuando es el periodo de atención alto, o sea, el ciclo durante el cual el cerebro es más eficiente, presto a procesar información. Estos periodos son denominados por los neurocientíficos, periodos básicos de vigilia-reposo (Salas, 2005).

\section{Matemáticas y emoción}

Muchos autores (Meyer y Turner, 2002; Schutz y Lanehart, 2002) enfatizan la relevancia que cada día ganan las emociones en el contexto educativo. Las emociones son inherentes al ser humano y muchas veces se manifiestan como una combinación letal para el aprendizaje a los niveles neurológicos, biológicos y psíquicos. Por lo anterior, el docente debe conocer la importancia que debe darle a las emociones, si desea obtener los mejores resultados en su tarea educativa o, de lo contrario, se convertirán en barreras de sus estrategias para el aprendizaje y la enseñanza. Es necesario mencionar que las emociones fomentan el aprendizaje cuando pueden estimular toda actividad a nivel de redes neuronales, intensificando las conexiones sinápticas. Por lo tanto, para la neurociencias (neurobiología) es mejor el aprendizaje cuando está involucrada la emoción (Immordino-Yang y Damasio, 2007).

La neurociencias cognitivas o neuropsicológicas son las que han contribuido más con sus investigaciones al contexto educativo tomando como variables las emociones. Un aspecto que destaca en estas investigaciones es el referido a las dos estructuras del sistema límbico: la amígdala y el hipocampo. Estas estructuras están relacionadas directamente con la corteza neofrontal. Si por alguna razón resultan inhibidas a causa del miedo o alguna situación de estrés, puede existir una disminución en el aprendizaje. Esta situación puede proyectarse sobre todo en el aprendizaje de las Matemáticas, disminuyendo la capacidad en la atención y motivación, afectando también el rendimiento.

Cuando una persona está bajo la acción de un evento emocional, la amígdala surge como centinela, dando la alarma e inicialmente se estimula la secreción de la hormona corticotropina (también neurotransmisor) y continúa con la secreción (a nivel suprarrenal) de cortisol. Esta última hormona disminuye su enfoque en la memoria de trabajo desviándolo hacia los sentidos. Cuando este evento emocional es prolongado (estrés a causa del miedo; por ejemplo), se cometen errores, la memoria falla, la atención es errática y aparece la dificultad de procesar información racionalmente.

Es necesario tener presente que elevados niveles de cortisol producen deterioro en células cerebrales, lo cual incide en la memoria y la atención al producir en la persona una baja en la autoestima y el rendimiento académico, así como una errática conducta para comunicarse (Conde, 2002).

La neurociencias ya ha demostrado fehacientemente que un evento emocional relevante (emoción traumática) se almacena en forma vivida e intensa y su recuerdo sobreexcita la amígdala, la cual reacciona inhibiendo otras acciones ejecutivas en el cerebro, como la atención y el razonamiento. 
Para LeDoux (1996), una vez que la estructura emocional ha aprendido, la tendencia es no olvidarlo, el aprendizaje se queda inmerso allí. Sin embargo, con una buena estrategia en que se aplique la psicoterapia, por ejemplo; se puede inducir al neocortex a disminuir la acción de la amígdala. Según Ledoux, esto ayudará al sujeto a superar la tendencia a reaccionar de forma automática, pero debe tenerse en cuenta que no desaparezcan la emociones básicas previamente producidas.

Phelps (2004), citado por Álvarez (2006), realizó un estudio sobre el funcionamiento de la amígdala en la extinción del miedo aprendido por condicionamiento. Los resultados fueron impresionantes. Hallaron que la eliminación sobre respuestas condicionadas no se debe a la supresión de las memorias de miedo, sino a un nuevo surgimiento en el aprendizaje o actividad memorística. Mencionan, además, que para la ocurrencia de este aprendizaje, la amígdala, así como la corteza ventral prefrontal, constituyen un nuevo circuito (memoria y aprendizaje), el cual entra en contienda con la memoria del miedo previamente establecida.

Álvarez (2006) también menciona que generalmente los miedos y la aversión a ciertos escenarios u objetos surgen, no porque se ha tenido una experiencia directa con el objeto o situación, sino que se han adquirido por leyendas, creencias, folclore cultural o experiencias negativas. Foss y Hadfield (1993) deducen que muchos estudiantes confrontan fobias desproporcionadas e irracionales hacia las Matemáticas.

\section{El aprendizaje matemático más allá del lenguaje y los símbolos}

Los docentes del futuro, sin lugar a dudas, soportarán su didáctica en las investigaciones relacionadas con las neurociencias. Un paradigma que surge con intensidad y marcará un hito tanto en la enseñanza como en el aprendizaje, especialmente en la enseñanza de las Matemáticas, es el enfoque denominado carácter multimodal de los conceptos, según Gallese y Lakoff (2005), citado por Radford et al. (2009). Este enfoque expresa que la actividad de pensar no solo es soportada por el lenguaje y los símbolos, sino que es inherente a la percepción sensorial. Gallese y Lakoff hacen énfasis en que el aprendizaje y enseñanza de las Matemáticas están muy relacionados con los sistemas sensorios y motor.

\section{Estrategias basadas en la neurociencias para el aprendizaje de las Matemáticas}

Algunas estrategias que surgen a partir de lo anteriormente escrito y analizado, se muestran a continuación:

- Las instituciones que imparten enseñanza deben considerar los aportes de la neurociencia hacia la educación. Es necesaria la formación del nuevo docente. Este nuevo docente debe reflexionar que la educación del futuro debe estar en armonía con el funcionamiento del cerebro y su estructura. Es lo que se ha llamado la enseñanza y el aprendizaje compatible con el cerebro.

Gairin (1991), citado por Guerrero (2004), menciona que la ansiedad matemática facilita el aprendizaje matemático de tareas complejas, en las circunstancias que se mencionan a continuación: a) cuando la autoestima no está amenazada, b) las tareas no se consideran 
novedosas o significativas, c) la ansiedad matemática es moderada o baja, y d) cuando el estudiante puede superar (a través de mecanismos cognitivos) esa ansiedad. El docente o la docente en matemáticas deben tener en consideración estas premisas en sus estrategias de enseñanza.

- Una estrategia importante, para el análisis y el razonamiento, es indudablemente el recurso de la memoria de trabajo. Por lo tanto, el docente debe promover actividades que estimulen la memoria de trabajo. Esto puede lograrse propiciando la aplicación de ejercicios para la memorización (listado serial numérico, lista telefónica, memorización de imágenes, lista de nombres), ejercicios sobre cálculo numérico, juegos analíticos, pensamiento lateral y acertijos entre otros. Tal como se describió anteriormente, la memoria de trabajo juega un papel trascendental, en la toma de decisiones, el razonamiento y el cálculo.

- La atención es otro recurso importante que involucra a la memoria de trabajo. Por eso, despertar la atención del estudiante es de vital importancia. El docente debe ser creativo e innovador para aumentar la motivación y estimular los niveles dopaminérgicos. A mayor concentración, menor es la ansiedad (en este caso, de la ansiedad matemática). Se debe evitar la inhibición, así como también el bloqueo en los alumnos. Este se presenta cuando la actividad atencional es débil y, como consecuencia, se pierde la oportunidad para utilizar el recurso de la memoria de trabajo en actividades que incluyan la resolución de problemas o las toma de decisiones.

- $\quad$ Jensen (2004) refiere que el cerebro enriquece su aprendizaje, cuando se le presentan retos en un entorno desafiante e interactuante, por ejemplo, la resolución de problemas. Esta estrategia, permitiría la generación de nuevas conexiones dendríticas y prepararía a los hemisferios cerebrales para alcanzar abstracciones complejas. Otra estrategia está referida al buen uso del lenguaje corporal o gestual.

La formación de ambientes que estimulen emociones positivas permitirá la activación dopaminérgica, como elementos claves en los procesos como vigilia y alerta. Es necesario enseñar a los estudiantes lo importante de su alimentación en su aprendizaje. Se les debe incentivar a consumir proteínas para potenciar la atención.

Resulta primordial observar que los neurotransmisores están disponibles en mayor cantidad durante el periodo matutino. Por lo tanto, el docente debe promover, en lo posible, los escenarios de aprendizajes en este periodo. De lo contrario, va a requerir de estrategias motivadoras y estimuladoras, para mantener el interés en sus clases.

Todo docente involucrado en la enseñanza de la Matemática conoce muy bien cómo influye el estrés en los estudiantes relacionados con el aprendizaje de esta asignatura. La fobia a las Matemáticas o ansiedad matemática, según lo mencionado por autores ya citados en este trabajo, es una constante que influye emocionalmente en la mayoría de los estudiantes y es la mayor causante del bajo rendimiento de estos, de su deterioro físico y psíquico, entre otros.

Por lo anterior, una estrategia de enseñanza en Matemáticas debe ir dirigida a técnicas de relajación. La relajación induce al sistema parasimpático a controlar el organismo, permitiendo distensión a nivel muscular y crear un ambiente tranquilo, así como estimulante. También el docente debe contribuir a desechar creencias, mitos y leyendas sobre el aprendizaje de las Matemáticas y mantener una actitud serena ante sus alumnos.

Además, el nuevo docente, consciente de la importancia que involucra las emociones en este nuevo paradigma educativo, debe despojarse de actitudes autoritarias e inflexibles. Autores como Waldegg han mencionado que es la emoción la puerta y la conexión directa al aprendizaje. Por lo 
tanto, estrategias tendientes a disminuir el estrés o fobia a las Matemáticas, un ambiente afectivo, colaborativo y cooperativo son relevantes en el aprendizaje.

Según el enfoque sobre el carácter multimodal, es necesario que el docente promueva estrategias de enseñanza enfocadas en la estimulación sensorio motor, porque, de acuerdo con este paradigma, induce al razonamiento en el calculo matemático. Sin olvidar jamás que la actividad para desarrollar el pensamiento tiene que estar acompañada de un verdadero desarrollo del lenguaje matemático y su correspondiente simbología.

\section{Conclusiones}

La investigación en neurociencia cognitiva ha demostrado que se avecinan cambios fundamentales en las ciencias de la educación. La integración de neurociencias y educación es inminente, y solo es necesaria la búsqueda de un diálogo fluido y continuo para lograr esta fusión. Se requiere la formación de un nuevo docente, formado por un currículo novedoso, para reformar un contexto estático y rígido donde educar se apoye en el aprendizaje compatible con el cerebro.

En la formación de este nuevo docente, el que enseña Matemáticas tendrá un papel preponderante. El viejo esquema del profesor autoritario, inflexible, con la convicción primaria de que solo alcanzan el éxito en esta asignatura los sujetos racionales, debe cambiar. Un docente en Matemáticas, cuyo criterio prevaleciente es: "racionalidad es igual a éxito", va a obtener un bajo porcentaje de éxitos en sus clases. Es un hecho fundamental y enfatizado ya por las neurociencias, el papel fundamental básico de la emocionalidad en la enseñanza y el aprendizaje.

Por lo tanto, ante este nuevo paradigma educativo, la formación del nuevo docente (a nivel básico y superior) debe incluir conocimientos en el aprendizaje compartible con el cerebro y su relación con las neurociencias cognitivas.

Se debe destacar la importancia que toma la memoria de trabajo en la enseñanza y aprendizaje del cálculo; pero también en otras disciplinas donde se requiera la participación en la toma de decisiones. Por ello el autor considera una línea de investigación dirigida a la memoria de trabajo y su relación a las Matemáticas y la resolución de problemas.

Uno de los obstáculos presentes en el desarrollo de las neurociencias, que impedirá una integración plena en la dinámica educativa es, sin duda, la falta de una base didáctica y las incoherencias o conflictos semánticos que ostenta. Por ejemplo, hay autores (Tenconi, 2006) que mencionan el desacuerdo marcado (semántico y didáctico) existente entre la neurociencias y la psiquiatría. Por ejemplo, cita este autor que al menos en algunos textos de neurociencias, neurobiología, neuroanatomía y neurofisiología, hay cinco o más formas para definir el sistema límbico y más de cinco formas de listar su estructura.

Si esto ocurre, en disciplinas tan íntimamente conexas, qué se puede esperar sobre las neurociencias cognoscitivas y la educación, disciplinas cuyas relaciones pedagógicas y didácticas apenas convergen.

Es significativo destacar la importancia que para el docente y la docente en Matemáticas resultan el conocer las bases y principios neurobiológicos que implican el funcionamiento cerebral, su desarrollo cognitivo, ontogenético y afectivo. Esto le permitirá tener en sus manos un gran recurso para diseñar su práctica docente, soportada sobre bases científicas. 


\section{Referencias bibliográficas}

Álvarez, J. (2006). Los hallazgos de las neurociencias y su aplicabilidad a la sala de clases: teoría y práctica. Puerto Rico: Ediciones Santillana.

Baddeley, A. (1997). Human Memory. Theory and Practice. New York: Mc Graw Hill.

Ballestra, M., Martínez, J. y Argibay, P. (2006, Agosto). Matemática y Cerebro. Revista del hospital italiano de Buenos Aires, 26(2), 79-84.

Bauselas, E. (2005, Octubre-Noviembre-Diciembre). Ansiedad ante los exámenes: evaluación e intervención psicopedagógica. Educere: La Revista Venezolana de Educación, Año 9. No. 31, 553-557.

Beilock, S. (2008). Math Performance in Stressful Situations [Rendimiento en Matemáticas bajo situaciones de Estrés]. Association for Psychological Science. The University of Chicago, 17(5), 339-343.

Bransford, J., Brown, A. y Cocking, R. (2003). How People Learn: Brain, Mind, Experience, and School. Estados Unidos: National Academy Press.

Carmona, S., Proal, E., Hoekzema, E. A., Gispert, J. D., Picado, M., Moreno, I., Soliva, J. C., Bielsa, A., Rovira, M., Hilferty, J., Bulbena, A., Casas, M., Tobeña, A. y Vilarroya, O. (2009, Noviembre). Ventro-Striatal reductions underpin symptoms of Hyperactivity and impulsivity in AttentionDeficit/Hyperactivity disorder [Reducciones Ventro-Estriadas corroboran los síntomas de hiperactividad e impulsividad en el trastorno de déficit de atención e hiperactividad]. Biol. Psychiatry, 66(10), 972-977.

Coleman, D. (2000). La Inteligencia Emocional. ¿Por qué es más importante que el cociente intelectual? (25 ed.). España: Editorial Vergara.

Conde, M. (2002). Estudios psicológicos. Revista de Psicología, 6(2), 112-122.

Covington, M. (2000, Febrero). Goal, theory, motivation, and school achievement: an integrative review [Objetivo de la teoría, la motivación y el rendimiento escolar: una revision integradora]. Annual Review of Psychology. 51, 171-200.

Edin, F., Klinberg, T., Johansson, P., McNab, F., Tegnér, J. y Compte, A. (2009, abril). Mechanism for top-down control of working memory capacity [Mecanismos para el control regulado de la memoria de trabajo]. Proceedings of the National Academy of Sciences [PNAS]. 106(16), 1-6, 6802-6807. Doi: 10.1073/pnas.0901894106

Foss, D. y Hadfield, O. (1993). A successful clinic for the reduction of mathematics anxiety among college students [Una aplicación clínica para la reducción de la ansiedad en matemáticas entre los estudiantes universitarios]. College Student Journal, 27(2), 157-166. 
Geake, J. G. (2005,Junio). The neurological basis of intelligence: Implications for education [Bases neurologicas de la inteligencia .Implicaciones para la Educacion]. Gifted \& Talented, 9(1), 8.

Goldin, G. (1988). Affective representation and mathematical problem solving. En M. J. Behr, C. B., Lacampagne; y M. M., Wheler (Eds.), Proceedings of the Tenth Annual Meeting on the Psychology of Mathematics Education, North American Chapter of International Group (pp. 1-7). DeKalb 10, Illinois: North Illinois University.

Guerrero, E. y Blanco, L. (2004, Julio). Diseño de un Programa Psicopedagógico para la Intervención en los Trastornos Emocionales en la Enseñanza y Aprendizaje de las Matemáticas. Revista Iberoamericana de Educación, 33(5), 1- 14.

Guisa, V. M., Riquelme, E. y San Vicente, G. (2007, Octubre). Memoria y Aprendizaje. Un modelo neurobiológico de las Adicciones. Conferencia Magistral del Congreso Internacional de Adicciones: Modelos de vanguardia para la atención. México, DF.

Immordino-Yang, M. y Damasio, A. (2007). We Feel, Therefore We Learn: The Relevance of Affective and Social Neuroscience to Education. Mind, Brain and Education, 1(1), 3-10.

Jensen, E. (2004). Cerebro y aprendizaje. Competencias e implicaciones educativas. Madrid: Ediciones Narcea S. A.

LeDoux, J. (1996). The Emotional Brain. New York: Simon \& Schuster.

Luria, A. (1988). El Cerebro en acción (5ª ed.). Barcelona: Editorial Martínez Roca.

Martínez, J. y Galán, F. (2000), Motivación, estrategias de aprendizaje y evaluación del rendimiento en alumnos universitarios. Iberpsicología, 5(2), 1-17.

McNab, F., Varrone, A., Farde, L., Jucaite, A., Bystritsky, P., Forssberg, H. \& Klingberg, T. (2009, Febrero). Changes in Cortical Dopamine D1 Receptor Binding Associated with Cognitive Training. Science. 323(5915), 800-802. Doi: 10.1126/science.1166102]

Meyer, D. y Turner, J. (2002). Discovering Emotion in Classroom Motivation Research. Educational Psychologist, 37(2) 107-114.

Morgado, I. (2005). Psicobiología del aprendizaje y la memoria. Cuadernos de Información y Comunicación, Vol. 10, 221-233.

Op't, E., De Corte., E. \& Verschaffel, L. (2001, August 28-September 1). Understanding the studentin context: What he feels, what he thinks, and what he does when solving a mathematical problem. Paper presented at the 9th EARLI-conference, in Fribourg, Switzerland.

Radford, L. y André, M. (2009, Julio). Cerebro, cognición y matemáticas. Revista latinoamericana de investigación en matemática educativa [RELIME], 12(2), 215-250. 
Rosselló, J. (1998). Psicología de la Atención. Madrid: Pirámide.

Salas, R. (2005). Neurociencia y educación. Cómo hacer una enseñanza más de acuerdo con la manera como aprende el cerebro. Valparaiso, Chile: Editorial Lafken Wangülen.

Schutz, P. y Lanehart, S. (2002, Spring). Emotions in Education [Emoción en la Educación]. Educational Psychologist. (Division Educational Psychology of the American Psychological Association), 37(2), 67-69.

Sprenger, M. (1999). Learning \& Memory. The Brain in Action. Virginia, USA: ASCD.

Tenconi, J. (2006). Tratado de Actualización en Psiquiatría. Buenos Aires: Editorial Sciens.

Volkow, N., Wang, G., Fowler, J., Telang, F., Maynard, L. y Logan J, (2004, Jul.). Evidence that methylphenidate enhances the saliency of a mathematical task by increasing dopamine in the human brain. The American Journal of Psychiatry, 161(7), 1173-1180.

Waldegg, G. (2003). Reseña al Libro La comprensión del cerebro. Hacia una nueva ciencia del aprendizaje. Educación Matemática, 15(003).

Wolfe, P. (2001). Brain Research and Education: Fad or Foundation? American Association of School Administrators, 63(11), 10-16. 